\title{
Orchestration of Tryptophan-Kynurenine Pathway, Acute Decompensation, and Acute-on-Chronic Liver Failure in Cirrhosis
}

\author{
Joan Clària (D) , ${ }^{1,2 *}$ Richard Moreau, ${ }^{1,3 *}$ François Fenaille, ${ }^{4}$ Alex Amorós,,${ }^{1}$ Christophe Junot, ${ }^{4}$ Henning Gronbaek, ${ }^{5}$ \\ Minneke J. Coenraad, ${ }^{6}$ Alain Pruvost, ${ }^{7}$ Aurélie Ghettas, ${ }^{7}$ Emeline Chu-Van, ${ }^{4}$ Cristina López-Vicario, ${ }^{2}$ Karl Oettl, ${ }^{8}$ Paolo Caraceni, ${ }^{9}$ \\ Carlo Alessandria, ${ }^{10}$ Jonel Trebicka (D) $1,11,12$ Marco Pavesi, ${ }^{1}$ Carme Deulofeu, ${ }^{1}$ Agustin Albillos, ${ }^{13}$ Thierry Gustot,${ }^{14}$ \\ Tania M. Welzel, ${ }^{12}$ Javier Fernández, ${ }^{1,2}$ Rudolf E. Stauber, ${ }^{8}$ Faouzi Saliba, ${ }^{15}$ Noémie Butin, ${ }^{4}$ Benoit Colsch, ${ }^{4}$ Christophe Moreno, ${ }^{14}$ \\ François Durand, ${ }^{3}$ Frederik Nevens, ${ }^{16}$ Rafael Bañares, ${ }^{17}$ Daniel Benten, ${ }^{18}$ Pere Ginès, ${ }^{2}$ Alexander Gerbes,,${ }^{19}$ Rajiv Jalan,${ }^{20}$ \\ Paolo Angeli, ${ }^{1,21}$ Mauro Bernardi (D) , 9 and Vicente Arroyo ${ }^{1}$; for the CANONIC Study Investigators of the EASL Clif Consortium, \\ Grifols Chair and the European Foundation for the Study of Chronic Liver Failure (EF Clif)
}

\begin{abstract}
Systemic inflammation (SI) is involved in the pathogenesis of acute decompensation (AD) and acute-on-chronic liver failure (ACLF) in cirrhosis. In other diseases, SI activates tryptophan (Trp) degradation through the kynurenine pathway (KP), giving rise to metabolites that contribute to multiorgan/system damage and immunosuppression. In the current study, we aimed to characterize the KP in patients with cirrhosis, in whom this pathway is poorly known. The serum levels of Trp, key KP metabolites (kynurenine and kynurenic and quinolinic acids), and cytokines (SI markers) were measured at enrollment in 40 healthy subjects, 39 patients with compensated cirrhosis, 342 with AD (no ACLF) and 180 with ACLF, and repeated in 258 patients during the 28-day follow-up. Urine KP metabolites were measured in 50 patients with ACLF. Serum KP activity was normal in compensated cirrhosis, increased in AD and further increased in ACLF, in parallel with SI; it was remarkably higher in ACLF with kidney failure than in ACLF without kidney failure in the absence of differences in urine KP activity and fractional excretion of KP metabolites. The short-term course of AD and ACLF (worsening, improvement, stable) correlated closely with follow-up changes in serum KP activity. Among patients with AD at enrollment, those with the highest baseline KP activity developed ACLF during follow-up. Among patients who had ACLF at enrollment, those with immune suppression and the highest KP activity, both at baseline, developed nosocomial infections during follow-up. Finally, higher baseline KP activity independently predicted mortality in patients with AD and ACLF. Conclusion: Features of KP activation appear in patients with AD, culminate in patients with ACLF, and may be involved in the pathogenesis of ACLF, clinical course, and mortality. (Hepatology 2019;69:1686-1701).
\end{abstract}

$\mathrm{T}$

The association of liver failure with extrahepatic organs/systems dysfunction is a characteristic feature of cirrhosis that affects

morbidity and mortality. In fact, acute-on-chronic liver failure (ACLF), which is characterized by systemic inflammation $(\mathrm{SI})$ and single or multiple $(\geq 2)$

Abbreviations: $A C L F$, acute-on-chronic liver failure; $A C L F-K F$, acute-on-chronic liver failure with kidney failure; $A D$, acute decompensation; a.u., arbitrary units; $B B B$, blood-brain barrier; BD, brain dysfunction; BF, brain failure; $C I$, confidence interval; HNA2, human nonmercaptalbumin 2; IDO, indoleamine 2,3-dioxygenase; IL, interleukin; $I Q R$, interquartile range; $K A$, kynurenic acid; KD, kidney dysfunction; $K F$, kidney failure; $K F$-free $A C L F$, acute-on-chronic liver failure without kidney failure; $K A$, kynurenic acid; $K P$, kynurenine pathway; $K Y N$, kynurenine; $L C-M S$, liquid chromatography-mass spectrometry; $M A P$, mean arterial pressure; NMDA, $N$-methyl-D-aspartic acid; $P C C$, plasma copeptin concentration; PRC, plasma renin concentration; $Q A$, quinolinic acid; SI, systemic inflammation; sMR/CD206, soluble macrophage mannose receptor 1; TDO, Tryptophan 2,3-dioxygenase; TNF $\alpha$, tumor necrosis factor $\alpha$; Trp, tryptophan; Trp-KYN derivatives, tryptophankynurenine derivatives; 3-HK, 3-hydroxykynurenine.

Received March 19, 2018; accepted October 22, 2018.

Additional Supporting Information may be found at onlinelibrary.wiley.com/doi/10.1002/hep.30363/suppinfo.

*These authors contributed equally to this work. 
organ failures, is the main cause of death in cirrhosis. ${ }^{(1)}$ The traditional paradigm of extrahepatic organ failure in cirrhosis relies on two principles: (1) They are functional disorders and (2) the pathogenesis is specific for each organ failure. However, this paradigm is changing and research has proven that SI and oxidative stress, which are well-known mechanisms of multiorgan failure in other clinical conditions, may be the common link between the diseased liver and extrahepatic organ failure in cirrhosis. ${ }^{(2)}$ Patients with decompensated cirrhosis exhibit chronic SI, possibly in relation to sustained intestinal bacterial translocation. In contrast, ACLF develops in the setting of further increase of SI promoted by precipitating factors (e.g., bacterial infections). Moreover, significant parenchymal renal and cerebral lesions and inflammatory changes have been described in kidney biopsies, brain autopsies, and imaging studies in patients with cirrhosis and experimental animals with renal failure or encephalopathy. ${ }^{(3-5)}$ Extrahepatic organ failure(s) in cirrhosis could therefore be the consequence of tissue immunopathology. ${ }^{(6)}$

SI also affects metabolism and induces changes in a myriad of biologically active small molecules. Among them, the degradation of tryptophan (Trp) through the kynurenine pathway (KP) to Trp-kynurenine derivatives ( $\mathrm{Trp}-\mathrm{KYN}$ derivatives) is of special interest in cirrhosis (Fig. 1). ${ }^{(7-9)}$ The KP is responsible for $95 \%$ of overall Trp degradation. The first and rate-limited step of KP is catalyzed by two enzymes: Tryptophan 2,3-dioxygenase (TDO) and indoleamine 2,3-dioxygenase (IDO). ${ }^{(8,9)}$ The expression of TDO2 (the gene-encoding TDO) is biased in the liver. In contrast, IDO1 and IDO2 (the genes encoding IDO) are predominantly extrahepatic and expressed primarily in peripheral blood immune cells, dendritic cells, endothelial cells, macrophages and microglia, astrocytes, and epithelial cells (e.g., renal tubular cells). ${ }^{(8,9)}$ Under

An appendix with the alphabetical list of CANONIC Study Investigators is provided.

Supported by the European Foundation for the Study of Chronic Liver Failure (EF-Clif). The EF-Clif is a non-profit private organization. Jonel Trebicka is an EF-Clif Visiting Professor. The EF-Clif receives unrestricted donations from Cellex Foundation and Grifols, and is partner or contributor in several EU Horizon 2020 program projects. The funders had no influence on study design, data collection and analysis, decision to publish or preparation of the manuscript.

(C) 2018 by the American Association for the Study of Liver Diseases.

View this article online at wileyonlinelibrary.com.

DOI 10.1002/hep.30363

Potential conflict of interest: Dr. Welzel consults, advises, and is on the speakers' bureau for AbbVie, Bristol-Myers Squibb, and Gilead. Dr. Durand consults and received grants from Gilead and Astellas; he consults for Bristol-Myers Squibb. Dr. Jalan is employed by and consults for Yaqrit.

\section{ARTICLE INFORMATION:}

From the ${ }^{1}$ European Foundation for the Study of Chronic Liver Failure Consortium and Grifols Chair, Barcelona, Spain; ${ }^{2}$ Hospital Clínic, IDIBAPS and CIBERehd, Barcelona, Spain; ${ }^{3}$ Inserm, Centre de Recherche sur l'Inflammation, Université Paris DiderotParis, Département Hospitalo-Universitaire UNITY; Service d'Hépatologie, Hôpital Beaujon, Assistance Publique-Hôpitaux de Paris; Laboratoire d'Excellence Inflamex, ComUE Sorbonne Paris Cité, Paris, France; ${ }^{4}$ CEA, INRA, Université Paris Saclay, Laboratoire d'Etude du Métabolisme des Médicaments, MetaboHUB-Paris, Gif-Sur-Yvette, France; ${ }^{5}$ Department of Hepatology \& Gastroenterology, Aarhus University Hospital, Aarhus, Denmark; ${ }^{6}$ Department of Gastroenterology and Hepatology, Leiden University Medical Center, Leiden, the Netherlands; ${ }^{7}$ CEA, INRA Université Paris Saclay, Service de Pharmacologie et Immunoanalyse, Plateforme SMArt-MS, Gif-sur-Yvette, France; ${ }^{8}$ Medical University of Graz, Graz, Austria; ${ }^{9}$ Department of Medical and Surgical Sciences, University of Bologna, Bologna, Italy; ${ }^{10}$ Division of Gastroenterology and Hepatology, San Giovanni Battista Hospital, Torino, Italy; ${ }^{11}$ Department of Internal Medicine I, University of Bonn, Bonn, Germany; ${ }^{12} \mathrm{~J} . W$. Goethe University Hospital, Frankfurt, Germany; ${ }^{13}$ Hospital Ramón y Cajal, Madrid, Spain; ${ }^{14}$ CUB Hopital Erasme, Université Libre de Bruxelles, Brussels, Belgium; ${ }^{15}$ Hôpital Paul Brousse, Université Paris-Sud, Villejuif, France; ${ }^{16}$ University Hospital Gasthuisberg, KU Leuven, Belgium; ${ }^{17}$ Facultad de Medicina, Universidad Complutense, Madrid, Spain; ${ }^{18}$ University Hospital Hamburg-Eppendorf, Germany; ${ }^{19}$ Department of Medicine II, University Hospital LMU Munich, Liver Center Munich, Munich, Germany; ${ }^{20}$ Liver Failure Group, Institute for Liver Disease Health, University College London, Royal Free Hospital, London, United Kingdom; ${ }^{21}$ Unit of Internal Medicine and Hepatology, Department of Medicine, DIMED, University of Padova, Padoa, Italy.

\section{ADDRESS CORRESPONDENCE AND REPRINT REQUESTS TO:}

Joan Clària

European Foundation for the Study of Chronic Liver Failure

Travessera de Gràcia 11, 08021 Barcelona, Spain
E-mail: joan.claria@efclif.com

Tel: +34-932-271-414 


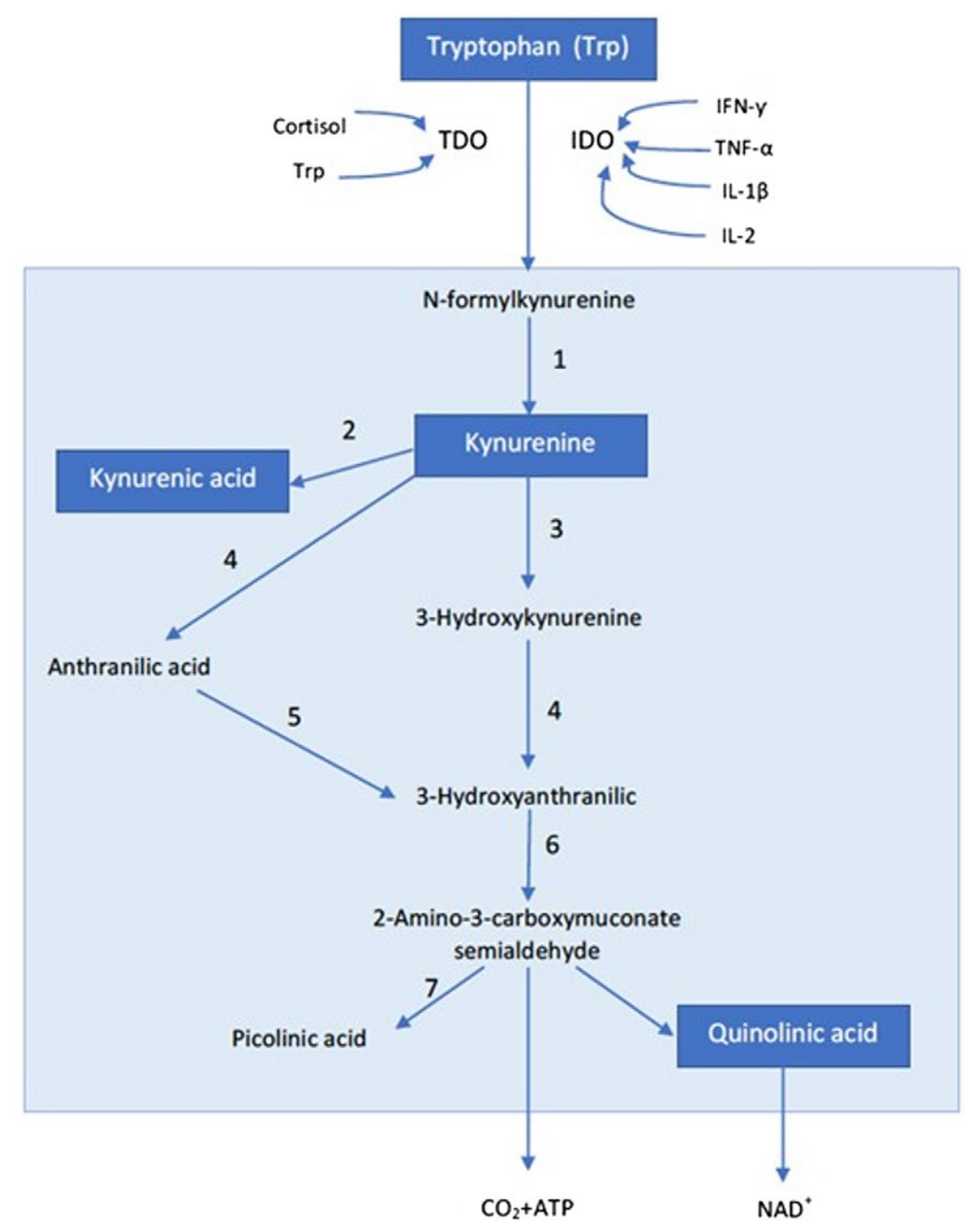

FIG. 1. Trp-KP metabolic pathway including potential stimuli of TDO and IDO. Numbers indicate the enzymes participating in each biosynthetic step: 1 = formamidase; 2 = kynurenine aminotransferase; 3 = kynurenine 3 -monooxygenase; 4 = kynureninase; 5 = anthranilate 3-monooxygenase; $6=3$-hydroxyanthranilic acid dioxygenase; and 7 = 2-hydroxymuconate-6-semialdehyde dehydrogenase. The molecules measured in the study are included in the blue boxes.

physiological conditions, most KP metabolites are constitutively synthesized within the liver by TDO; only a marginal proportion is synthesized outside the liver. However, in the setting of SI there is intense overexpression of IDO and extrahepatic production of $\operatorname{Trp}-\mathrm{KYN}$ derivatives including kynurenine (KYN, which is an endothelial-derived relaxing factor and neuroactive molecule), quinolinic acid (QA, which is a neuronal $N$-methyl-D-aspartic acid [NMDA] receptor neurotoxic agonist), and kynurenic acid (KA, which is a NMDA antagonist). KYN and KA exert immunomodulatory actions by binding to aryl hydrocarbon receptor and GPR35 in immune cells. ${ }^{(9,10)}$ In addition, the KP can produce picolinic acid (a second NMDA receptor antagonist), as well as the immunosuppressive metabolites 3-hydroxykynurenine (3-HK) and 3-hydroxyanthranilic acid (3-HAA). ${ }^{(7-9)}$ Finally, during $\mathrm{KP}$ activation, there is increased generation of highly reactive oxygen and nitrogen species, which contribute to immunopathology. IDO expression can be increased by interferon $\gamma$, tumor necrosis factor $\alpha$ ( TNF $\alpha$ ), and other cytokines.

Circulating Trp can be transported across the cell membrane and the blood-brain barrier (BBB) only in 
its free form, which represents approximately 5\% of the total Trp concentration in plasma. ${ }^{(11)}$ The remaining molecules circulate bound to plasma albumin. In patients with decompensated cirrhosis, however, free Trp in plasma is increased due to hypoalbuminemia, structural changes in the albumin molecule, and competition with endogenous and exogenous substances for the binding to albumin Sudlow site II, ${ }^{(12)}$ including kynurenines. Once within the cells, TDO and IDO interact with $\operatorname{Trp}$ and initiate the KP. Although the complete set of enzymes of the KP is expressed only in the liver, kynurenines can cross the cellular membrane through L-type amino acid transporter, which is also used by Trp, and act in extrahepatic cells as intermediate substrates for the synthesis of downstream metabolites. ${ }^{(13)}$ Metabolites of the KP, therefore, may act as autocrine, paracrine, and even as endocrine mediators.

Kynurenines are involved in the pathogenesis of diseases associated with acute and chronic SI, including severe sepsis, acute pancreatitis, metabolic syndrome, acute confusional states, depression, and chronic neurodegenerative diseases. Because the level of KP activation is poorly known in cirrhosis, this observational, prospective study was aimed to investigate the potential role of $\mathrm{KP}$ activity in acute decompensation (AD) and ACLF in cirrhosis.

\section{Experimental Procedures}

\section{STUDY POPULATION}

The study was performed in patients included in the CANONIC study, an European multicenter, prospective, observational investigation in 1343 consecutive patients with cirrhosis hospitalized for the treatment of $\mathrm{AD}$. In this study, clinical data and bio samples were obtained not only at enrollment (which generally coincided with hospital admission), but also sequentially during a follow-up period of 28 days. ${ }^{(1)}$

This study included 342 randomly selected patients with AD and 180 with ACLF at enrollment (95 ACLF-1, 65 ACLF-2, and 19 ACLF-3). In all patients $(n=522)$, the serum KP (which is mentioned as KP throughout the study) was assessed at enrollment and in 258 measurements that were repeated at the last hospital visit within the 28-day follow-up period. The urine KP was assessed in 50 patients with ACLF. A detailed description of data and sample collection policy in the CANONIC study is given in the Supporting Information. A diagram of the patients included in the current study is shown in Supporting Fig. S1. All patients included have participated in other investigations; therefore, many data have been reported previously. ${ }^{(14-17)}$

Thirty-nine additional nonhospitalized patients with compensated cirrhosis (no previous history of $\mathrm{AD})$ and 40 healthy blood donors (age: 45-65 years) were studied as controls. All patients with compensated cirrhosis had clinically significant portal hypertension, as indicated by presence of esophageal varices and/or high FibroScan liver stiffness.

Details regarding the institutional research boards that approved the CANONIC study and the current study are found in the Supporting Information.

\section{MEASUREMENT OF Trp AND Trp-KYN DERIVATIVES BY LIQUID CHROMATOGRAPHY COUPLED WITH MASS SPECTROMETRY}

Trp and Trp-KYN derivatives in serum were first assessed in the frame of an untargeted liquid chromatography-mass spectrometry (LC-MS)-based metabolomics approach. Metabolites were extracted from serum samples following methanol-assisted protein precipitation. Therefore, detected metabolites were in their free state, not bound to albumin and/or other proteins. Extracts were analyzed by LC-MS using an Ultimate 3000 chromatographic system (Thermo Fisher Scientific, Courtaboeuf, France) coupled with an Exactive mass spectrometer (Thermo Fisher Scientific) fitted with an electrospray source and operating in the positive and negative ion modes for metabolite separations on C18 and ZIC-pHILIC columns, respectively. ${ }^{(18)}$ The metabolite concentrations from the different experimental batches were standardized using the LOESS algorithm. ${ }^{(19)}$ Absolute targeted quantification was performed in 234 randomly selected subset of samples from the whole group (16 healthy subjects, 20 patients with compensated cirrhosis, 100 with $\mathrm{AD}$, and 98 with ACLF). Serum extracts were injected into a LC-MS/MS system consisting of a Waters ACQUITY UPLC system (Milford, MA) equipped with an UPLC charged surface hybrid column and coupled with a Waters 
XEVO TQ-XS mass spectrometer operating in the positive ion electrospray multiple reaction monitoring mode. Quantification was performed using standard calibration curves and internal labeled standards ( $\mathrm{d}_{5}-\mathrm{L}-\mathrm{Trp},{ }^{13} \mathrm{C}_{6}-\mathrm{L}-\mathrm{KYN} \mathrm{d}_{5}-\mathrm{KA}$, and ${ }^{13} \mathrm{C}_{3},{ }^{15} \mathrm{~N}_{1}$-QA $)$. To explore the renal handling of Trp and KYN derivatives in patients with ACLF, absolute serum and urine concentrations were measured by LC-MS/MS in 50 randomly selected samples from patients with ACLF (25 with kidney failure and 25 without kidney failure). Plasma and urine creatinine levels were also measured by a standard method based on Jaffé's reaction, and the fractional renal excretion of each individual metabolite was calculated based on the equation ([urinary TrpKYN derivative concentration/serum Trp-KYN derivative concentration]/[urinary creatinine concentration/ serum creatinine concentration] x 100). More detailed information is given in the Supporting Information.

\section{OTHER MEASUREMENTS}

\section{Assessment of SI and Systemic Oxidative Stress}

SI was assessed by the plasma levels of 17 cytokines and the circulating markers of macrophage activation soluble CD163 (sCD163) and mannose receptor $(\mathrm{sMR} / \mathrm{sCD} 206)$. Oxidative stress was assessed through the analysis of the redox state of plasma albumin (human nonmercaptalbumin 2 [HNA2]). These results have been previously reported. ${ }^{(15,16)}$

\section{Biomarkers of Systemic Circulatory Function}

Plasma copeptin concentration (PCC) and plasma renin concentration (PRC), which estimate antidiuretic hormone release and the activity of the renin-angiotensin system, respectively, were selected as biomarkers of circulatory function.

\section{Assessment of IDO1 and IDO2 Gene Expression in Peripheral Blood Cells}

IDO1 and IDO2 messenger RNA (mRNA) expression was determined by real-time PCR in RNA from peripheral blood mononuclear cells (PBMCs) isolated from healthy volunteers $(n=4)$ and patients with $A D$ cirrhosis $(\mathrm{n}=8)$ (see Supporting Information).

\section{DEFINITIONS}

The following definitions concerning clinical features are detailed in the Supporting Information: compensated and decompensated cirrhosis, $\mathrm{AD}$, ACLF, organ failures including liver failure, kidney failure $(\mathrm{KF})$, brain failure $(\mathrm{BF})$, coagulation failure, circulatory failure and respiratory failure, kidney dysfunction (KD), brain dysfunction (BD), ACLF grades, and clinical courses of $\mathrm{AD}$ and ACLF.

\section{STATISTICAL ANALYSIS}

Results are presented as frequency and percentage for categorical variables, mean, and SD for normally distributed continuous variables, and median and interquartile range for not normally distributed continuous variables. More details are found in the Supporting Information.

\section{Results}

\section{"BEDSIDE" DATA AND "BENCH" RESULTS INVESTIGATING SYSTEMIC CIRCULATORY FUNCTION AND SI AT ENROLLMENT}

Age and gender were similar in the three groups of patients (compensated cirrhosis, AD, and ACLF) (Supporting Table S1). The prevalence of alcoholic cirrhosis increased and the prevalence of cirrhosis associated with hepatitis $\mathrm{C}$ virus ( $\mathrm{HCV}$ ) decreased progressively across the three groups of patients with cirrhosis. There were significant differences among patients with compensated cirrhosis, AD, and ACLF in standard liver and renal function tests, platelets, C-reactive protein, and white blood count. The most frequent complication associated with cirrhosis at enrollment in patients with AD and ACLF was ascites, followed by encephalopathy, bacterial infections, and gastrointestinal bleeding.

PCC, PRC, and mean arterial pressure (MAP) were normal in patients with compensated cirrhosis, markedly increased (PCC and PRC) or decreased (MAP) in patients with $\mathrm{AD}$, and significantly more deteriorated in patients with ACLF (Supporting Table S2). PCC, which was more sensitive than PRC 
in differentiating $\mathrm{AD}$ from $\mathrm{ACLF}$, was used for association analysis. The plasma levels of cytokines were normal or only moderately increased in patients with compensated cirrhosis, markedly elevated in patients with $\mathrm{AD}$, and significantly higher in patients with ACLF than in patients with AD (Supporting Table S2). Plasma sCD163 and sMR/sCD206 levels were increased in patients with $\mathrm{AD}$ and significantly higher in patients with ACLF than in patients with AD. Finally, plasma levels of HNA2 were increased to a similar degree in patients with compensated and decompensated cirrhosis, but significantly higher in patients with ACLF.

\section{KP IS ACTIVATED IN PATIENTS WITH AD AND ACLF}

During the assessment of the whole serum metabolome using an untargeted and semi-quantitative HRMS-based approach, we detected four major components of the KP (Trp, KYN, KA, and QA) at sufficiently high levels to permit accurate and reliable measurements. KYN, KA, and $\mathrm{QA}$ are among the most relevant kynurenines and are produced at different steps of the KP (Fig. 1). Accordingly, measuring the serum levels of these metabolites provides comprehensive information on KP activity. In addition, the KYN to $\operatorname{Trp}$ ratio (KYN/Trp) is used widely to estimate the TDO and IDO activity. ${ }^{(20)}$ Table $1 \mathrm{~A}$ lists the serum levels of these metabolites, as measured by LC-MS and expressed in relative units corresponding to chromatographic peak areas, in healthy controls and patients with compensated cirrhosis, $\mathrm{AD}$, and ACLF. In patients with compensated cirrhosis, the KP activity was not significantly different from healthy controls. In contrast, there were major changes in patients with AD and ACLF. KYN/Trp (Table 1A) and serum levels of KYN, KA, and QA (Table 1A, Fig. 2A) were higher, whereas Trp levels were lower (Table 1A, Fig. 2B), in patients with $\mathrm{AD}$ and ACLF relative to patients with compensated cirrhosis and healthy controls, which is consistent with increased Trp degradation and enhanced production of kynurenines due to overactivity of TDO or IDO. Indeed, mRNA expression for both IDO1 and IDO2 was significantly up-regulated in $\mathrm{PBMC}$ from patients with $\mathrm{AD}$ with respect to healthy donors (IDO1: $6.2 \pm 2.7$ versus 0.40 \pm 0.1 arbitrary units [a.u.], $P<0.05 ;$ IDO2: $1.9 \pm 0.5$ versus $0.3 \pm 0.2$ a.u., $P<0.03)$. Interestingly, patients with ACLF had the lowest levels of Trp, and the highest KYN/Trp and serum levels of KYN, KA, and QA. KA and QA were the most sensitive markers of $\mathrm{KP}$ activation in our patients, and therefore were used thereafter for most association analyses.

Table $1 \mathrm{~B}$ indicates that these differences in the serum levels of Trp and selected Trp-KYN derivatives between groups were similarly observed in the subset of 218 patients in whom absolute concentrations were measured using synthetic standards during the LC-MS analysis. All values detected in the serum were within the $\mathrm{ng} / \mathrm{mL}$ to $\mu \mathrm{g} / \mathrm{mL}$ range. The correlation coefficients between both types of measurements were Trp: $r=0.91$;YN: $r=0.77$; KA: $r=0.87$; QA: $\mathrm{r}=0.93 ; P<0.0001$ for all.

\section{KP ACTIVATION CORRELATES WITH SI INTENSITY}

There were significant but weak positive correlations between inflammatory mediators and serum levels of $\mathrm{KA}$ and $\mathrm{QA}$ in the whole series of patients (Supporting Table S3), suggesting an association among SI, IDO, and KP overactivity. The strongest association was observed between $\mathrm{QA}$ and TNF $\alpha$ (Fig. 2C).

\section{KP ACTIVITY AND OVERALL SEVERITY OF CIRRHOSIS HAVE SIMILAR ORCHESTRATION}

$\mathrm{KP}$ overactivity is associated with impairment of systemic circulatory function. Although KP activity rose progressively across patient groups of increasing severity (Table $1 \mathrm{~A}$ ), there were parallel progressive decline in MAP and increase in PCC and PRC (Supporting Table S2). Moreover, KA and QA levels had significant direct correlations with PCC ( $\mathrm{r}=$ $0.39 ; P<0.001$ and $\mathrm{r}=0.48 ; P<0.001$, respectively). Together these findings show a positive correlation between the intensity of impairment in circulatory function and the KP activity.

\section{Hierarchization of the KP Activity According to the Existence of Kidney or Brain Failure or Dysfunction}

Among patients with ACLF at enrollment, those who had kidney failure (ACLF-KF) exhibited 


\section{TABLE 1. Metabolites of the Kynurenine Pathway at Enrollment, Across Different Groups of Individuals}

\begin{tabular}{|c|c|c|c|c|c|}
\hline Groups of individuals & Tryptophan (Trp) & Kynurenine (KYN) & KYN/Trp ratio & Kynurenic acid & Quinolinic acid \\
\hline A. All study individuals & \multicolumn{5}{|c|}{ 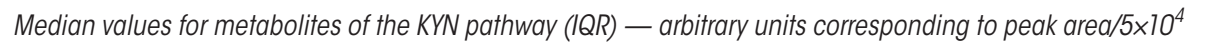 } \\
\hline Healthy subjects $(n=40)$ & $469(390-590)$ & $6.4(3.6-8.0)$ & $0.012(0.008-0.016)$ & $0.64(0.50-0.89)$ & $11.1(7.7-28.9)$ \\
\hline Compensated cirrhosis $(n=39)$ & $512(429-554)$ & $7.7(6.2-25.5)$ & $0.017(0.012-0.050)$ & $0.82(0.46-1.00)$ & $11.8(7.7-22.9)$ \\
\hline$A D(n=337)$ & $372(255-527)$ & $9.8(5.8-21.6)$ & $0.026(0.014-0.061)$ & $0.86(0.49-1.44)$ & $24.5(16.0-47.2)$ \\
\hline $\operatorname{ACLF}(n=180)$ & $353(240-489)$ & $15.2(7.5-28.3)$ & $0.038(0.022-0.092)$ & $2.26(1.06-6.66)$ & $96.2(30.2-184.4)$ \\
\hline$P$ value & $<0.001$ & $<0.001$ & $<0.001$ & $<0.001$ & $<0.001$ \\
\hline B. Subsets of study individuals & \multicolumn{5}{|c|}{ Median values for metabolites of the $K P(I Q R)(\mu \mathrm{g} / \mathrm{mL})$} \\
\hline Healthy subjects $(n=16)$ & $10.45(9.37-13.37)$ & $0.32(0.26-0.37)$ & $0.026(0.024-0.034)$ & $0.0048(0.0042-0.0059)$ & $0.046(0.042-0.052)$ \\
\hline Compensated cirrhosis $(n=20)$ & $12.82(11.47-14.20)$ & $0.51(0.46-0.66)$ & $0.047(0.033-0.054)$ & $0.006(0.05-0.0081)$ & $0.083(0.056-0.12)$ \\
\hline$A D(n=100)$ & $9.48(6.37-13.83)$ & $0.49(0.35-0.61)$ & $0.049(0.039-0.068)$ & $0.007(0.004-0.01)$ & $0.15(0.08-0.24)$ \\
\hline $\operatorname{ACLF}(n=98)$ & $8.96(6.48-11.81)$ & $0.75(0.53-0.98)$ & $0.081(0.061-0.113)$ & $0.018(0.009-0.039)$ & $0.42(0.17-0.79)$ \\
\hline \multirow[t]{2}{*}{$P$ value } & 0.0016 & $<0.001$ & $<0.001$ & $<0.001$ & $<0.001$ \\
\hline & \multicolumn{5}{|c|}{ 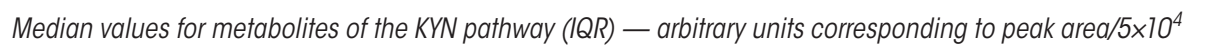 } \\
\hline \multicolumn{6}{|l|}{$\begin{array}{l}\text { C. Patients with either AD, ACLF-KF, or } \\
\text { KF-free ACLF }\end{array}$} \\
\hline$A D(n=337)$ & $372(255-527)$ & $9.8(5.8-21.6)$ & $0.026(0.014-0.061)$ & $0.86(0.49-1.44)$ & $24.5(16.0-47.2)$ \\
\hline KF-free ACLF $(n=76)$ & $381(250-562)$ & $9.9(5.6-19.1)$ & $0.025(0.013-0.049)$ & $1.14(0.50-2.29)$ & $39.9(22.2-105.1)$ \\
\hline ACLF-KF $(n=103)$ & $332(224-435)$ & $19.5(11.3-35.1)$ & $0.057(0.030-0.119)$ & $4.82(1.99-11.92)$ & $131.2(70.5-255.3)$ \\
\hline$P$ value & 0.050 & $<0.001$ & $<0.001$ & $<0.001$ & $<0.001$ \\
\hline \multicolumn{6}{|l|}{$\begin{array}{l}\text { D. Patients with } A D \text { according to the } \\
\text { presence or absence of brain dysfunc- } \\
\text { tion (BD) or kidney dysfunction (KD) }\end{array}$} \\
\hline No BD/No KD $(n=209)$ & $376(258-541)$ & $8.2(5.2-16.3)$ & $0.021(0.013-0.046)$ & $0.68(0.43-1.07)$ & $21.1(13.4-40.3)$ \\
\hline$B D(n=60)$ & $411(293-549)$ & $13.9(7.4-31.8)$ & $0.032(0.015-0.086)$ & $0.99(0.63-1.54)$ & $26.8(18.3-43.1)$ \\
\hline$K D(n=38)$ & $337(198-437)$ & $16.6(9.7-28.9)$ & $0.050(0.025-0.091)$ & $1.89(1.27-3.06)$ & $55.8(31.6-99.5)$ \\
\hline $\mathrm{BD}$ and $\mathrm{KD}(\mathrm{n}=21)$ & $316(298-436)$ & $21.7(6.9-36.8)$ & $0.050(0.023-0.109)$ & $1.51(0.73-2.30)$ & $45.6(20.1-77.0)$ \\
\hline$P$ value & 0.291 & $<0.001$ & $<0.001$ & $<0.001$ & $<0.001$ \\
\hline \multicolumn{6}{|l|}{$\begin{array}{l}\text { E. Patients stratified according to their } \\
\text { clinical course by } 28 \text { days }\end{array}$} \\
\hline AD throughout the study $(n=281)$ & $376(253-541)$ & $9.6(5.6-21.1)$ & $0.025(0.014-0.059)$ & $0.79(0.46-1.28)$ & $23.4(15.6-44.6)$ \\
\hline Development of KF-free ACLF $(n=23)$ & $383(312-507)$ & $10.7(5.8-16.9)$ & $0.022(0.012-0.062)$ & $1.11(0.60-1.67)$ & $31.3(16.4-48.2)$ \\
\hline Development of ACLF-KF $(n=33)$ & $332(258-443)$ & $12.1(7.1-29.4)$ & $0.042(0.021-0.091)$ & $1.48(0.88-2.25)$ & $45.6(20.6-97.2)$ \\
\hline$P$ value & 0.452 & 0.154 & 0.045 & 0.001 & $<0.001$ \\
\hline
\end{tabular}

Note: $P$ values are from the overall comparisons.

AD denotes acute decompensation; KF, kidney failure; ACLF-KF, ACLF associated with KF; KF-free ACLF, ACLF not associated with KF.

lower baseline serum levels of Trp and higher KYN, $\mathrm{KA}$, and QA than those who had KF-free ACLF (Table 1C, Fig. 2A,B). These data indicate that KP overactivity is more marked in patients with $\mathrm{KF}$ than in those who had an ACLF form defined by the presence of extrarenal organ failures. Of note, the serum levels of KA and QA were higher in patients with $\mathrm{KF}$-free ACLF than in those with $\mathrm{AD}$, indicating that KF-free ACLF is also associated with KP overactivity, although to a lesser degree than in the case of ACLF-KF. Interestingly, among patients with $\mathrm{AD}$, there was higher $\mathrm{KP}$ activity in patients with $\mathrm{KD}$ relative to patients free of both kidney and brain dysfunctions (Table 1D), indicating that KP overactivity is also higher in patients with $\mathrm{AD}$ and $\mathrm{KD}$ than in those without $\mathrm{KD}$.

We performed additional analyses to explore the intrarenal handling of KP metabolites in the context of ACLF. For this, we measured the serum and urine creatinine and absolute serum and urine Trp and KYN, $\mathrm{KA}$, and $\mathrm{QA}$ concentrations and the renal fractional excretion of these metabolites in 25 patients with ACLF-KF and 25 with KF-free ACLF (Table 2). In agreement with our first results expressed in relative units (Table 2), the results of additional experiments confirmed that the absolute serum concentrations 
A
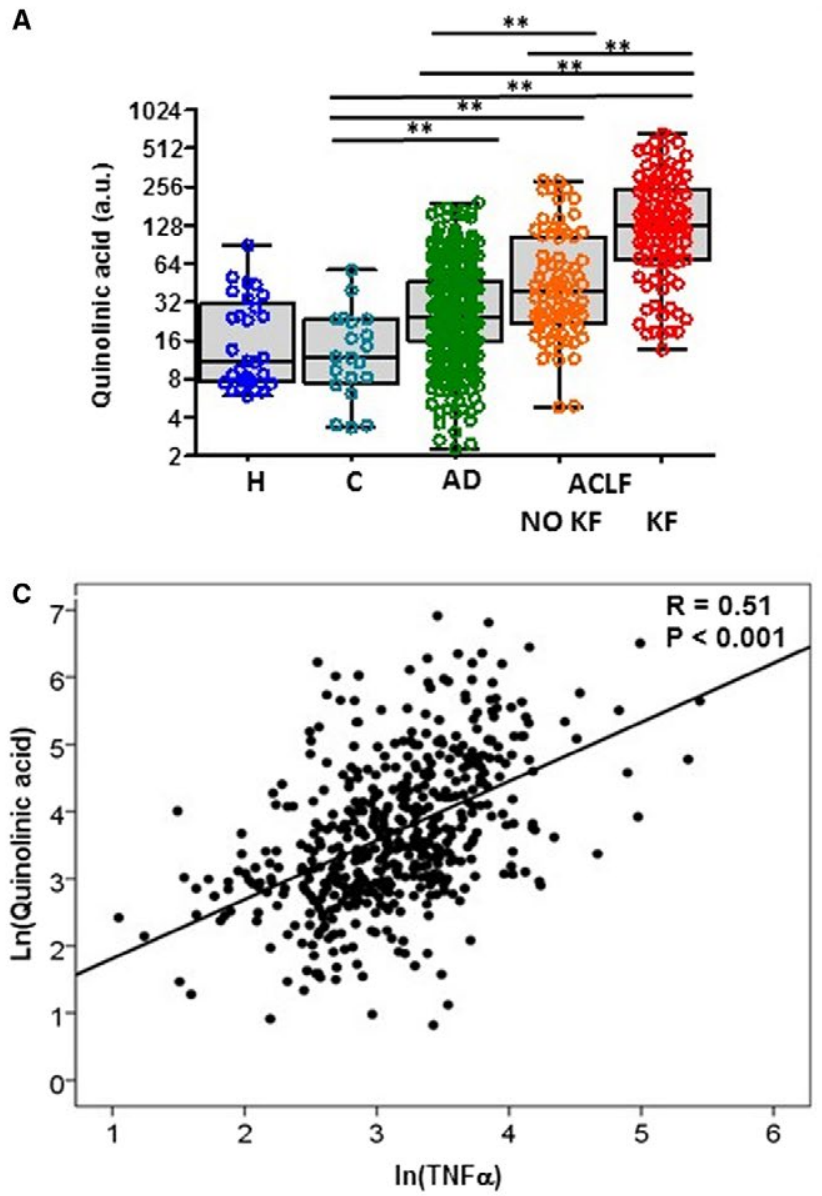

B
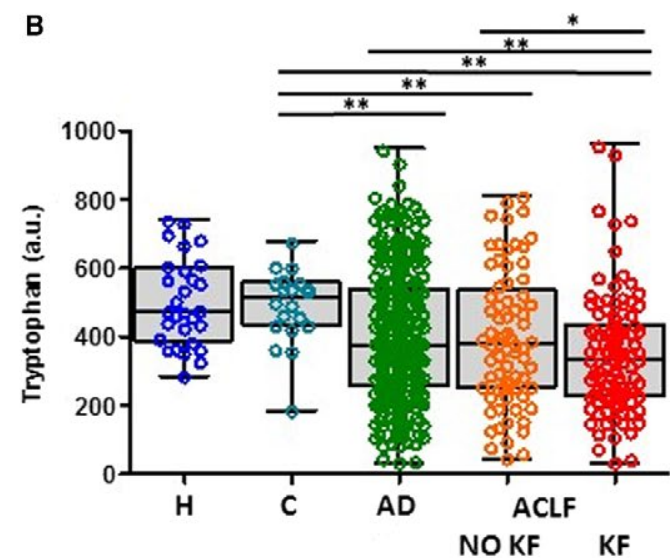

D
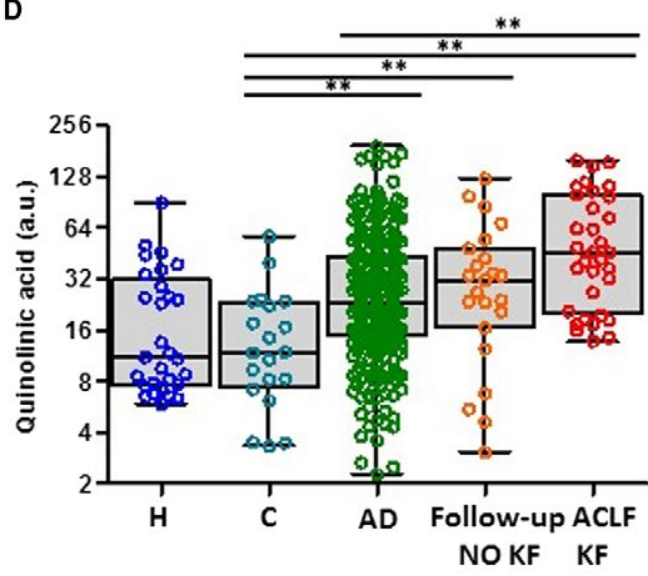

FIG. 2. (A) Serum levels of $Q A$ at enrollment in healthy subjects $(H)$ and patients with compensated cirrhosis (C), AD, and ACLF without and with kidney failure (No KF and KF), respectively. (B) Serum levels of Trp at enrollment in healthy subjects (H) and patients with compensated cirrhosis $(\mathrm{C}), \mathrm{AD}$, and $\mathrm{ACLF}$ without and with kidney failure (No KF and KF, respectively). (C) Correlation between QA and TNF $\alpha$. (D) Serum levels of QA at enrollment in healthy subjects (H), patients with compensated cirrhosis (C), patients with AD throughout the study, and patients with baseline AD who developed ACLF without and with kidney failure (No KF and KF, respectively) during follow-up. The values in (A) and (D) are represented in quadratic scale and in (C) in natural logarithmic scale. Because the serum levels of QA were similar in healthy subjects and compensated cirrhosis, statistical comparison among groups in $(A),(B)$, and (D) were performed between the groups of patients with cirrhosis. ${ }^{*} P<0.05 ;{ }^{* *} P<0.001$. Values in the metabolites of the $\mathrm{KP}$ are in arbitrary units corresponding to peak area/5 $\times 10^{4}$.

of $\mathrm{KYN}, \mathrm{KA}$, and QA were increased significantly in patients with $\mathrm{KF}-\mathrm{ACLF}$ as compared with those with KF-free ACLF. In contrast, the urinary concentration and the fractional urinary excretion of these Trp-KYN metabolites were similar in the two groups of patients (Table 2). There was yet marked heterogeneity in the urine-to-serum ratio of the levels of each Trp-KYN metabolite, within each group of patients. For example, although the urine-to-serum ratios for $\mathrm{KA}$ concentrations was +472 and +88 in patients with $\mathrm{KF}$-free ACLF and those with ACLF-KF, respectively, the ratios were lower for QA $(+116$ and +19 , respectively) or even much lower for KYN (+3.3 and -0.66 fold, respectively). The urine concentration of Trp was markedly lower in patients with ACLF-KF than in patient with KF-free ACLF.

There were also close associations between KP overactivity and hepatic encephalopathy. Most patients (36 of 43 patients) with BF had ACLF, and therefore very high levels of KA (1.82 [0.90-4.39] a.u.) and QA (67.2 [26.5-156.6] a.u.). The serum levels of KA and $\mathrm{QA}$ were also higher in the 60 patients with $\mathrm{AD}$ and $\mathrm{BD}$ alone than in the 209 patients without $\mathrm{KD}$ and/ or BD (Table 1D). 
TABLE 2. Serum and Urine Concentrations of Tryptophan, Kynurenine, Kynurenic Acid, and Quinolinic Acid in Patients with ACLF, According to the Presence or Absence of Kidney Failure

\begin{tabular}{|c|c|c|c|}
\hline \multirow[b]{2}{*}{ Variable } & \multirow{2}{*}{$\frac{\text { No Kidney Failure }}{(\mathrm{N}=25)}$} & \multirow{2}{*}{$\begin{array}{c}\text { Kidney Failure } \\
(\mathrm{N}=25)\end{array}$} & \multirow[b]{2}{*}{$P$-value } \\
\hline & & & \\
\hline \multicolumn{4}{|l|}{ Serum } \\
\hline Median values for creatinine levels (IQR) (mg/mL) & $0.009(0.007-0.010)$ & $0.030(0.023-0.036)$ & $<0.001$ \\
\hline \multicolumn{4}{|c|}{ Median values for metabolites of the KYN pathway (IQR) $(\mu \mathrm{g} / \mathrm{mL})$} \\
\hline Tryptophan & $10.70(5.95-12.41)$ & $10.76(7.68-13.78)$ & 0.7562 \\
\hline Kynurenine & $0.65(0.41-0.85)$ & $0.96(0.52-1.33)$ & 0.0117 \\
\hline Kynurenic acid & $0.007(0.005-0.013)$ & $0.028(0.017-0.036)$ & $<0.001$ \\
\hline Quinolinic acid & $0.15(0.10-0.27)$ & $0.55(0.42-0.78)$ & $<0.001$ \\
\hline \multicolumn{4}{|l|}{ Urine } \\
\hline Median values for creatinine levels (IQR) (mg/mL) & $0.658(0.575-1.002)$ & $0.540(0.424-0.687)$ & 0.0727 \\
\hline \multicolumn{4}{|c|}{ Median values for metabolites of the KYN pathway (IQR) $(\mu \mathrm{g} / \mathrm{mL})$} \\
\hline Tryptophan & $14.19(7.52-25.31)$ & $5.53(3.28-13.20)$ & 0.0071 \\
\hline Kynurenine & $2.24(0.97-2.97)$ & $0.68(0.35-2.26)$ & 0.0547 \\
\hline Kynurenic acid & $3.31(2.01-3.90)$ & $3.27(1.83-4.46)$ & 0.8766 \\
\hline Quinolinic acid & $13.33(10.21-19.41)$ & $10.71(5.94-21.89)$ & 0.4151 \\
\hline \multicolumn{4}{|c|}{ Median values for renal fractional excretion of metabolites (\%) } \\
\hline Tryptophan & $1.8(0.94-2.8)$ & $3.3(1.8-7.8)$ & 0.0135 \\
\hline Kynurenine & $3.2(1.6-5.5)$ & $4.5(2.9-10.7)$ & 0.1511 \\
\hline Kynurenic acid & $439.6(335.4-566.3)$ & $519.2(339.2-804.7)$ & 0.2523 \\
\hline Quinolinic acid & $104.4(78.0-125.9)$ & $98.9(90.3-153.3)$ & 0.6554 \\
\hline
\end{tabular}

Together these findings indicate that, in patients with $\mathrm{AD}$ and in those with ACLF, the degree of KP activation is related closely to the presence of alterations in kidney and brain functions, and to their intensities.

\section{KP Overactivity Is Higher in Patients With Ongoing Bacterial Infections Than in Those Without}

As expected, plasma cytokines ${ }^{(16)}$ and markers of $\mathrm{KP}$ activity (serum levels of $\mathrm{KA}$ and $\mathrm{QA}$ ) were significantly higher in patients with bacterial infections at enrollment than in patients without, both in the whole series of patients (Supporting Table S4) and among patients with ACLF (Supporting Table S5).

\section{Different Clinical Courses of AD and ACLF Are Associated With Distinct Profiles of KP Activity}

There was a close association between changes in the serum levels of KA and QA and the 28-day follow-up clinical course of patients with $\mathrm{AD}$ and ACLF (Table 3). Improvement of ACLF (36 patients with ACLF-1, 30 with ACLF-2, and 3 with ACLF-3) and worsening of $\mathrm{AD}$ or ACLF (25 patients with no ACLF, 10 with ACLF-1, and 11 with ACLF-2) were associated with parallel changes in the serum levels of KA and QA. Patients with AD steady course and those with ACLF steady course (22 patients had ACLF-1, 15 had ACLF-2, and 11 had ACLF-3) did not present significant changes in the serum levels of KA and QA during the 28-day follow-up.

\section{ANTECEDENCE OF INCREASED KP ACTIVITY RELATIVE TO THE DEVELOPMENT OF ACLF}

Among the 337 patients with AD at enrollment, those who developed ACLF-KF during the 28-day follow-up period had higher baseline KA and QA concentrations than those who developed KF-free ACLF (Table 1E, Fig. 2D). The baseline concentrations of these kynurenines were also higher in patients developing KF-free ACLF relative to patients without ACLF throughout the study (Table 1, Fig. 2D), although differences were not statistically significant. Our findings therefore show an antecedence of an increase in $\mathrm{KP}$ activity relative to the development of ACLF-KF. 
TABLE 3. Metabolites of the KP at Enrollment and Last Follow-Up Assessment Among Groups of Patients Who Differed in Their Clinical Course (Improvement, Worsening, Steady State)

\begin{tabular}{|c|c|c|c|c|}
\hline Patient Groups & $\mathrm{n}$ & Enrollment & Last Assessment & $P$ Value \\
\hline \multicolumn{5}{|c|}{ Median values for Trp levels (IQR) (a.u. correspond to peak area/5 $\times 10^{4}$ ) } \\
\hline Improvement of ACLF & 69 & $395(251-507)$ & $397(291-509)$ & 0.560 \\
\hline ACLF steady course & 48 & $355(235-473)$ & $354(216-547)$ & 0.952 \\
\hline Worsening of $\mathrm{AD}$ or $\mathrm{ACLF}$ & 46 & $330(228-492)$ & $369(244-569)$ & 0.307 \\
\hline \multirow[t]{2}{*}{ AD steady course } & 95 & $379(272-634)$ & $430(291-645)$ & 0.258 \\
\hline & \multicolumn{4}{|c|}{ Median values for KYN levels (IQR) (a.u. correspond to peak area/5 $\times 10^{4}$ ) } \\
\hline Improvement of ACLF & 69 & $12.5(6.1-29.1)$ & $10.8(6.5-23.5)$ & 0.412 \\
\hline ACLF steady course & 48 & $17.7(9.8-32.8)$ & $14.0(8.3-22.9)$ & 0.058 \\
\hline Worsening of $\mathrm{AD}$ or $\mathrm{ACLF}$ & 46 & $13.5(7.3-27.6)$ & $13.9(8.1-18.0)$ & 0.203 \\
\hline \multirow[t]{2}{*}{ AD steady course } & 95 & $9.8(5.2-17.4)$ & $8.7(5.3-15.3)$ & 0.543 \\
\hline & \multicolumn{4}{|c|}{ Median values for the KYN to Trp ratio (IQR) } \\
\hline Improvement of ACLF & 69 & $0.031(0.020-0.064)$ & $0.029(0.017-0.067)$ & 0.372 \\
\hline ACLF steady course & 48 & $0.048(0.024-0.117)$ & $0.045(0.015-0.091)$ & 0.214 \\
\hline Worsening of $A D$ or ACLF & 46 & $0.043(0.020-0.093)$ & $0.032(0.024-0.066)$ & 0.081 \\
\hline \multirow{2}{*}{ AD steady course } & 95 & $0.021(0.012-0.054)$ & $0.019(0.012-0.037)$ & 0.096 \\
\hline & \multicolumn{4}{|c|}{ Median values for KA levels (IQR) (a.u. correspond to peak area/5 $\times 10^{4}$ ) } \\
\hline Improvement of ACLF & 69 & $1.60(0.93-2.84)$ & $0.84(0.48-1.47)$ & $<0.001$ \\
\hline ACLF steady course & 48 & $5.37(1.90-10.39)$ & $3.62(1.05-18.28)$ & 0.310 \\
\hline Worsening of AD or ACLF & 46 & $1.63(0.88-2.94)$ & $2.36(1.15-4.68)$ & 0.009 \\
\hline \multirow[t]{2}{*}{ AD steady course } & 95 & $0.77(0.49-1.13)$ & $0.64(0.45-0.97)$ & 0.509 \\
\hline & \multicolumn{4}{|c|}{ Median values for $Q$ A levels (IQR) (a.u. correspond to peak area $\left./ 5 \times 10^{4}\right)$} \\
\hline Improvement of ACLF & 69 & $60.5(29.9-120.2)$ & $43.7(22.6-79.9)$ & $<0.001$ \\
\hline ACLF steady course & 48 & $127.3(50.7-272.5)$ & $104.8(48.4-246.8)$ & 0.896 \\
\hline Worsening of $\mathrm{AD}$ or $\mathrm{ACLF}$ & 46 & $53.9(25.0-117.7)$ & $89.0(49.9-159.1)$ & 0.023 \\
\hline AD steady course & 95 & $22.7(16.4-45.2)$ & $27.5(15.8-43.1)$ & 0.126 \\
\hline
\end{tabular}

\section{ANTECEDENCE OF INCREASED KP ACTIVITY RELATIVE TO THE DEVELOPMENT OF ACLF-RELATED INFECTIOUS COMPLICATIONS}

As expected, ${ }^{(17)}$ a high proportion (56\%) of the 108 patients who had ACLF but no infection at enrollment subsequently developed bacterial infection during the 28-day follow-up. Of note, none of the baseline characteristics obtained at bedside with the exception of serum albumin could predict the development of bacterial infection (Supporting Table S6). In contrast, at baseline, several "bench" values assessing SI intensity were higher in patients who developed infection during follow-up than in those who did not develop this complication (Fig. 3A, Table 4). Moreover, significantly higher baseline levels of signals for immune suppression (i.e., interleukin [IL]-10 [Fig. 3B] and sMR/sCD206) were detected. Among the variety of SI markers evaluated at baseline, only IL-10 and
sMR/sCD206 were independently associated with follow-up bacterial infections in patients with ACLF who were "uninfected" at enrollment (odds ratio [95\% confidence interval (CI)]): Log (IL-10), 1.35 (1.04-1.73), $P=0.022 ; \log (\mathrm{sMR} / \mathrm{sCD} 206), 2.80$ (1.13-6.90), $P=0.026)$. The area under receiver operating characteristic curves for predicting the development of infection was 0.695 (95\% CI, 0.595-0.796) for IL-10 and 0.679 (95\% CI, 0.575-0.784) for sMR/sCD206. In addition, the combination of these two markers of immunosuppression $(-0.083+0.296 \ln [\mathrm{IL}-10]+1.028 \ln [\mathrm{sMR} /$ sCD206]) significantly predicted the risk of bacterial infections (Fig. 3C). Together these findings support the existence of immune suppression in patients with $\mathrm{ACLF}$, providing a rational explanation for the high risk of developing bacterial infection in these patients. ${ }^{(17)}$ Because KP activation can cause immune suppression through different mechanisms, ${ }^{(21)}$ we compared, among patients with ACLF and no infection at enrollment, the baseline levels of KP metabolites of patients who developed infection during follow-up with those 
A
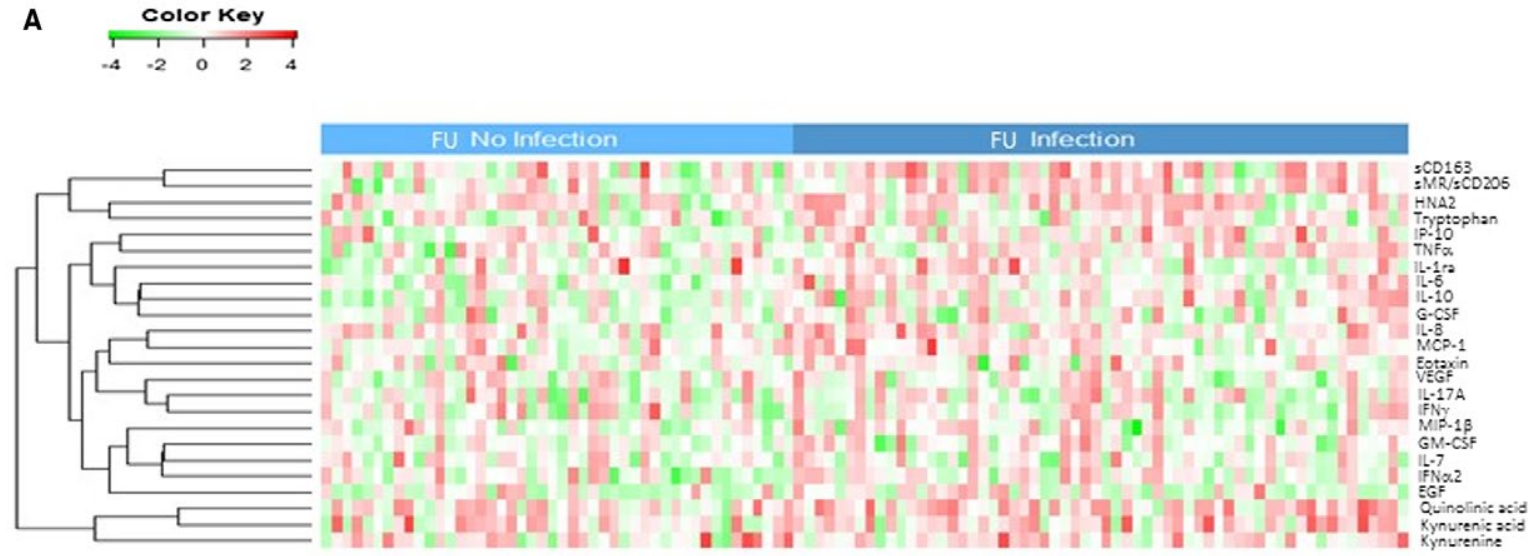

B

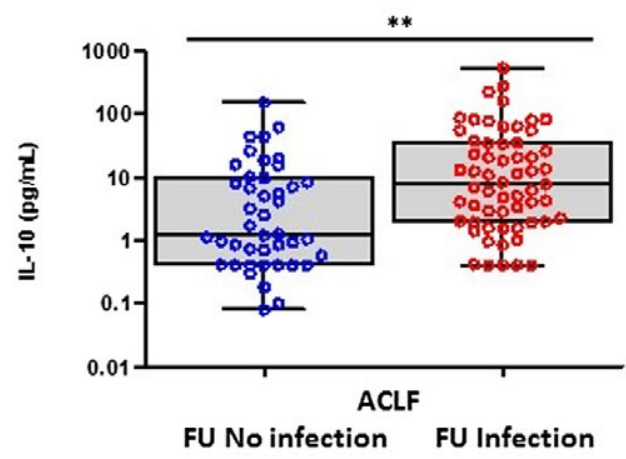

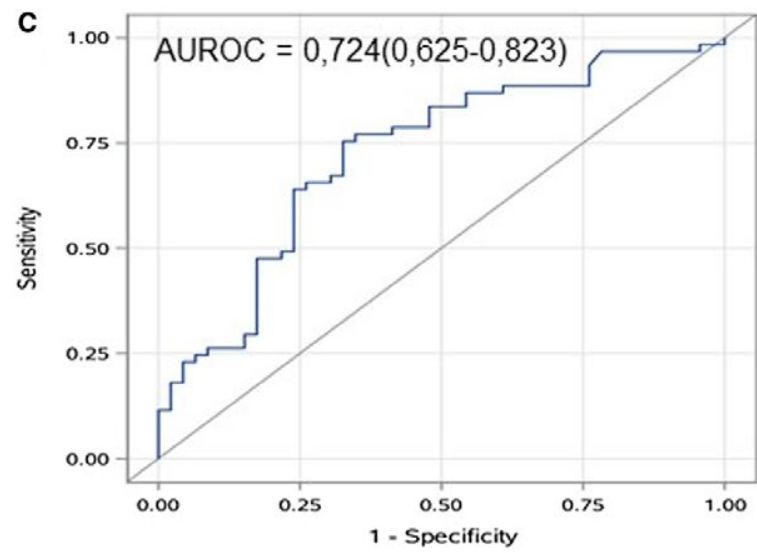

FIG. 3. (A) Differential baseline patterns of inflammatory mediators/markers and metabolites of the KP in ACLF patients who did or did not develop follow-up bacterial infections. The pattern identifies higher markers of SI, immunosuppression (sMR/sCD206 and IL-10), and KP activity in patients who developed infections during FU. (B) Individual baseline values of IL-10 in ACLF patients who did or did not develop follow-up bacterial infections (values represented in quadratic scale). ${ }^{* *} P<0.001$. (C) Accuracy of combining the baseline plasma levels of IL-10 and sMR/sCD206 in predicting 28-day follow-up bacterial infections in "uninfected" patients with ACLF. Abbreviations: AUROC, area under the receiver operating characteristic curve; FU, follow-up.

patients who did not develop this complication. Thus, serum QA levels were higher in patients who developed infections during follow-up (Table 4), indicating that among patients with ACLF, an increase in KP activity precedes the onset of nosocomial bacterial infection.

\section{KP OVERACTIVITY IS AN INDEPENDENT PREDICTOR OF SURVIVAL IN CIRRHOSIS}

Based on results of univariate analysis (Supporting Tables S7 and S8), multivariate analysis identified 11 independent baseline predictors of 90-day mortality in the whole series of patients (Table 5). Among these predictors, there were only four variables obtained at bedside, including older age, higher bilirubin, higher international normalized ratio, and lower serum sodium and 7 "bench" variables, including higher levels of KYN, IL-8, endothelial growth factor, vascular endothelial growth factor, and sMR/sCD206, and lower levels of Trp and INF $\gamma$. Based on results of the univariate analysis (Supporting Tables S9 and S10), we identified four independent baseline predictors of 28-day mortality in patients with ACLF: older age, higher bilirubin, higher $\mathrm{KYN}$, and higher sCD163 (Table 5). Together, these findings indicate that increased KP activity is a major predictor of poor outcome in patients with $\mathrm{AD}$ and in those with ACLF. 
TABLE 4. Plasma Cytokines, Biomarkers of Macrophage Activity and Systemic Inflammation, and KP Metabolites in Patients With ACLF, Without Infection at Enrollment, Who Did and Did Not Develop Infection During 28-Day Follow-up

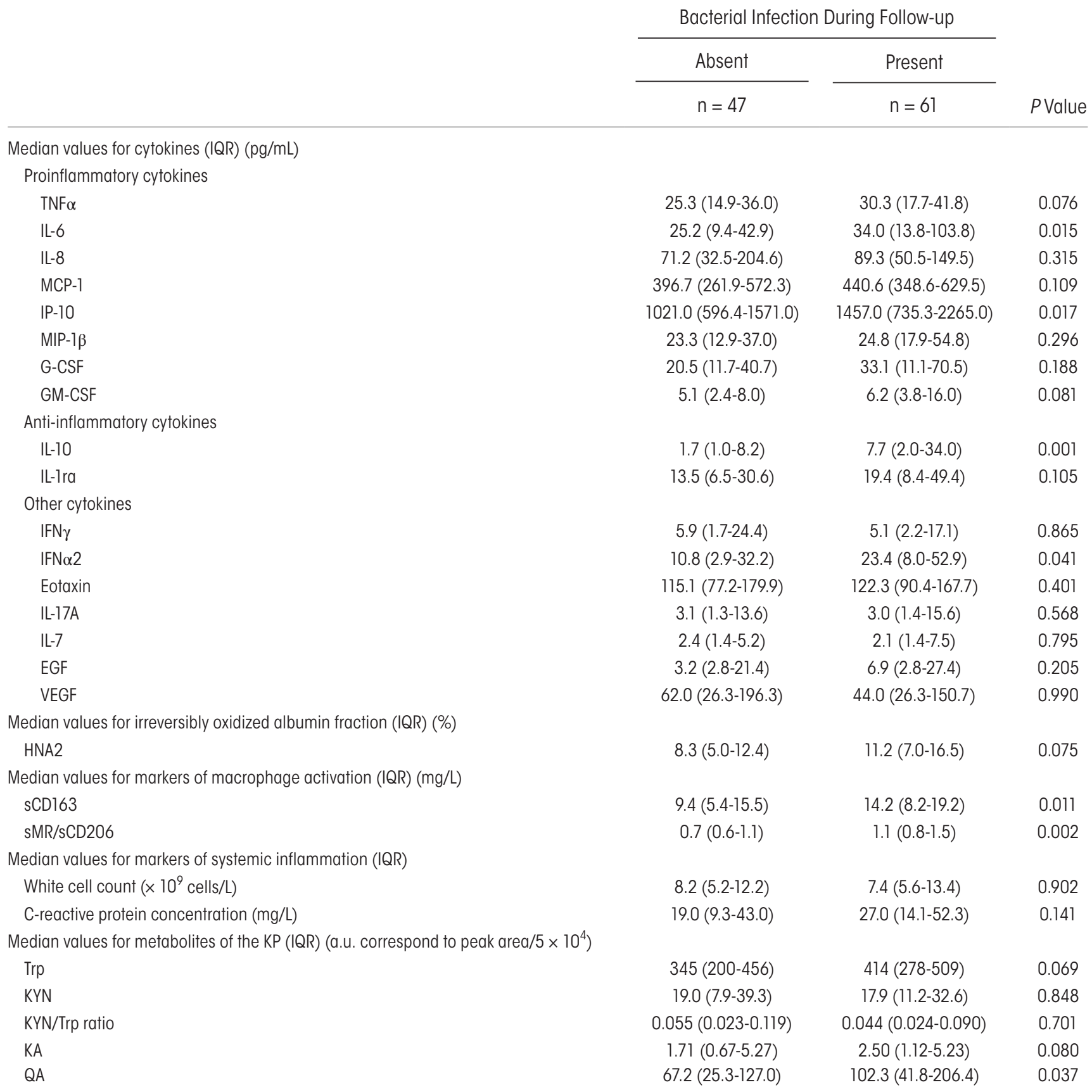

Abbreviations: EGF, epidermal growth factor; G-CSF, granulocyte colony-stimulating factor; GM-CSF, granulocyte-macrophage colony-stimulating factor; IL-1ra, interleukin-1 receptor antagonist protein; IP-10, $10 \mathrm{kD}$ interferon gamma-induced protein; MCP1 , monocyte chemotactic protein 1; MIP-1ß, macrophage inflammatory protein 1-beta; VEGF, vascular endothelial growth factor.

\section{Discussion}

There are several investigations showing activation of KP in plasma, cerebrospinal fluid and brain tissue in primary biliary cirrhosis, $\mathrm{HCV}$-associated chronic hepatitis, cirrhosis, hepatocellular carcinoma, and hepatic encephalopathy. ${ }^{(22-25)}$ Our study is a prospective observational investigation to assess Trp degradation through the KP in a large series of patients with cirrhosis at different stages of the disease and its 
TABLE 5. Independent Baseline Predictors of 90-Day Mortality in the Whole Series of Patients and 28-Day Mortality in Patients With ACLF

Whole Series of Patients

\begin{tabular}{|c|c|c|c|c|}
\hline \multirow[b]{2}{*}{ Variable } & & \\
\hline & HR for Death at 90 days ( $95 \% \mathrm{Cl})$ & PValue & HR for Death at 28 days ( $95 \% \mathrm{Cl}$ ) & $P$ Value \\
\hline $\operatorname{Ln}(\operatorname{Trp})$ & $0.71(0.53-0.97)$ & 0.029 & - & \\
\hline $\operatorname{Ln}(K Y N)$ & $1.36(1.14-1.62)$ & $<0.001$ & $1.66(1.29-2.15)$ & $<0.001$ \\
\hline $\operatorname{Ln}(I L-8)$ & $1.26(1.00-1.58)$ & 0.047 & - & \\
\hline $\operatorname{Ln}(\mathrm{IFN} \gamma)$ & $0.82(0.71-0.95)$ & 0.008 & - & \\
\hline $\operatorname{Ln}(E G F)$ & $0.85(0.72-0.99)$ & 0.041 & - & \\
\hline Ln(VEGF) & $1.29(1.10-1.51)$ & 0.001 & - & \\
\hline Age & $1.04(1.02-1.06)$ & $<0.001$ & $1.06(1.03-1.09)$ & $<0.001$ \\
\hline Ln(Bilirubin) & $1.32(1.04-1.70)$ & 0.025 & $1.77(1.23-2.56)$ & 0.002 \\
\hline $\operatorname{Ln}(\mathrm{INR})$ & $2.90(1.62-5.18)$ & $<0.001$ & - & \\
\hline $\operatorname{Ln}(\mathrm{sCD} 163)$ & - & & $2.07(1.12-3.83)$ & 0.020 \\
\hline Ln(sMR/sCD206) & $2.36(1.42-3.91)$ & $<0.001$ & - & \\
\hline Serum sodium & $0.96(0.93-1.00)$ & 0.033 & - & \\
\hline
\end{tabular}

Abbreviations: EGF, epidermal growth factor; HR, hazard ratio; INR, international normalized ratio; Ln, natural logarithm; VEGF, vascular endothelial growth factor.

relationship with the severity of the disease, clinical course, and survival. We report six important findings. First, KP activity was normal in patients with compensated cirrhosis but markedly increased in patients with $\mathrm{AD}$ and particularly in those with ACLF. Second, our results were consistent with activation of $\mathrm{KP}$ as a result of SI. Third, KP activity and overall severity of cirrhosis followed similar orchestration. Indeed, among patients with $\mathrm{AD}, \mathrm{KP}$ activity was higher in patients with $\mathrm{KD}$ or $\mathrm{BD}$ than in those without these dysfunctions. Among patients with ACLF, KP activity was remarkably higher in patients with ACLF-KF than in patients with KF-free ACLF. Fourth, we found an antecedence of increased $\mathrm{KP}$ activity relative to the development of ACLF. Fifth, we observed an antecedence of $\mathrm{KP}$ overactivity relative to ACLF-related infectious complications. Finally, higher KP activity was an independent predictor of death in patients with $\mathrm{AD}$ (at 90 days) and in those with ACLF (at 28 days).

The first issue to be discussed is the strong association between KF and high circulating levels of Trp-KYN derivatives in patients with ACLF, which has been proposed to be related to a reduction in the renal excretion of Trp-KYN derivatives and not to the mechanism of kidney failure. Our data do not support this contention. Indeed, the urinary concentration of $\mathrm{KYN}, \mathrm{KA}$, and $\mathrm{QA}$ were similar in our patients with KF-free ACLF and in those with ACLF-KF despite marked differences in serum concentrations of
Trp-KYN derivatives. Moreover, the fractional excretion of KYN, KA and QA, which estimates the percentage of the filtered Trp-KYN derivatives excreted by the urine, were also similar between groups. Therefore, the difference in the levels of circulating Trp-KYN derivatives between patients with ACLF and $\mathrm{KF}$ and those without $\mathrm{KF}$ cannot be explained by differences in the renal excretion of $\operatorname{Trp}-\mathrm{KYN}$ derivatives.

The heterogeneity of urine $\operatorname{Trp}-\mathrm{KYN}$ derivative profiles in patients with ACLF was a key observation that may help to understand the potential mechanism of the extremely higher activation of the KP associated with ACLF-KF relative to KF-free ACLF, the absence of differences between the urine levels of the Trp-KYN derivatives in both conditions, the mechanism of the specific urine profile of each Trp-KYN derivative, and the pathogenesis of the marked activation of the renal KP in patients with ACLF-KF.

Whereas the urine-to-serum ratios for KA concentrations were strikingly increased in both groups of patients with ACLF, the increases in ratios for QA concentrations, although important, were significantly less marked, and the increases in ratios for KYN concentrations were either mild or inexistent. These differences in the urine-to-serum ratios between TrpKYN derivatives were poorly related with glomerular filtration rate (as estimated by serum creatinine) and their corresponding serum levels. Together, these 
findings strongly suggest a tubular mechanism (e.g., tubular secretion) that is unrelated to renal dysfunction as the most likely explanation for high urine-toserum ratios of some Trp-KYN metabolites, but not all.

The renal tubular cells are among the most sensitive cells for expressing the Trp-consuming enzyme IDO in response to inflammatory cues. ${ }^{(26)}$ Here, we found that the urine concentration of Trp was markedly lower in patients with ACLF-KF than in those with KF-free ACLF, suggesting that Trp consumption is related to higher degree of renal inflammation in the former group of patients. Therefore, it is likely that in patients with ACLF-KF, kidney inflammation could lead to increased tubular release of Trp-KYN derivatives into the urine and perhaps also to spillover in the systemic circulation. The differences in profile of Trp-KYN derivatives in serum and urine probably reflects differences in their origin (primarily the tubular cells in the urine and from many different types of cells, including the tubular cells, in serum). The development of $\mathrm{KF}$ and associated tubular release of Trp-KYN derivatives would depend on the severity of renal inflammation. This feature was detected in serum, but not in urine, probably because the severity of renal failure impairs the mechanisms influencing the tubular release of endogenous metabolites, especially the tubular urine flow rate.

Kynurenines have deleterious effects on brain function and participate in the pathogenesis of a variety of acute and chronic brain disorders and diseases associated with SI. ${ }^{(13,27)}$ Because the expression of IDO in the brain is low, tissue concentration of kynurenines depends largely on the transport of circulating kynurenines across the BBB. KYN and 3-HK, but not KA and QA, are readily transported across the $\mathrm{BBB}$. Once in the brain, they are further degraded to produce KA and QA and other kynurenines. QA and, with less intensity, 3-HK and 3-HAA have neurotoxic effects due to their capacity to generate reactive oxygen species. QA also causes neuronal excitotoxicity through the activation of NMDA (glutamate) receptors. The ultimate consequences of these mechanisms are brain inflammation and oxidative stress, changes in neurotransmission, and neuronal dysfunction and apoptosis.

There is evidence from both clinical and experimental studies that brain inflammation and hyperammonemia are major mechanisms of hepatic encephalopathy. ${ }^{(28)}$ There are some preliminary data suggesting that activated KP may also play a contributory role. Two-fold to 10 -fold elevations of cerebrospinal fluid QA concentrations have been reported in 4 neonates with hepatic coma and in 6 patients with cirrhosis who died with hepatic encephalopathy. ${ }^{(24,25)}$ The cortical content of QA was also 3 times higher in the latest group of patients than in a control group of patients with cirrhosis dying from other causes. In addition, high levels of KYN in cerebrospinal fluid and plasma have recently been reported in patients with cirrhosis and hepatic encephalopathy. Our data showing a close association between high KP activity and the presence of $\mathrm{BD}$ and $\mathrm{BF}$ in patients with $\mathrm{AD}$ and $\mathrm{ACLF}$, respectively, are in keeping with this suggestion. The neurotoxic effects of QA and other kynurenines could also explain the surprisingly extended areas of gray and white matter losses described in patients with decompensated cirrhosis. ${ }^{(5)}$

KYN has been identified as an endothelium-derived relaxing factor, the release of which is promoted by the effect of the inflammatory mediators on IDO, leading to activation of soluble guanylyl cyclase in the underlying arteriolar smooth muscle cells and vasodilation. ${ }^{(29)}$ Inhibition of IDO activity protects animals with sepsis from hypotension, indicating that $\mathrm{KP}$ is a major contributory mechanism of systemic circulatory dysfunction in SI. ${ }^{(30)}$ Our results suggest that this may also be the case in patients with cirrhosis with $\mathrm{AD}$ and $\mathrm{ACLF}$, as the circulating levels of $\mathrm{QA}$ and other kynurenines correlated directly with the degree of systemic circulatory dysfunction. Angiotensin II also increases the expression of IDO, activates the $\mathrm{KP}$, and promotes oxidative stress and apoptosis in endothelial cells. ${ }^{(30)}$ This mechanism may be important in patients with cirrhosis with ACLF, because the renin-angiotensin system is markedly activated in these patients. Therefore, the sequential occurrence of acute and severe SI, increased endothelial IDO and KP activity and oxidative stress, KYN mediated arteriolar vasodilation, homeostatic stimulation of the renin-angiotensin system, and further increase in the $\mathrm{KP}$ activity and oxidative stress represents a potential vicious pathophysiological circle that could lead to progressive endothelial cell apoptosis, microcirculatory dysfunction, and organ failure.

The KYN/Trp ratio and the plasma levels of kynurenines are accurate predictors of multiorgan failure and mortality in critically ill patients hospitalized 
by acute BF of different etiologies, ${ }^{(31)}$ severe trauma, ${ }^{(32)}$ acute pancreatitis ${ }^{(33)}$ and sepsis, ${ }^{(34)}$ suggesting that KP activity is a critical determinant in the evolution of diseases associated with acute SI. In fact, the blockade of the two key enzymes of the KP, IDO, and KMO reduces the rate of multiorgan failure and mortality in experimental sepsis or acute pancreatitis. ${ }^{(30,35,36)}$ Our results indicate that the course of ACLF correlated closely with changes in plasma KA, QA, and Trp concentrations, and that kynurenines were independent predictors of mortality both in patients with $\mathrm{AD}$ and with ACLF. This supports the idea that KP activity might be an important mechanism of multiorgan failure and mortality in cirrhosis.

Fernández et al. recently showed that patients hospitalized with ACLF not triggered by infections are highly predisposed to develop nosocomial infections, suggesting immune paralysis. ${ }^{(17)}$ Our data support this contention, as the higher circulating levels of $\mathrm{IL}-10$ and $\mathrm{sMR} / \mathrm{sCD} 206$ in patients with $\mathrm{ACLF}$ were the only independent predictors of nosocomial infections in these patients. The activation of IDO is a potent immunosuppressive mechanism ${ }^{(10,37,38)}$ and increases IL-10 release. Because the baseline values of $\mathrm{KP}$ activity were also higher in patients with ACLF developing nosocomial infections than in those who did not develop infections, our data might indicate that kynurenines contribute to the immunosuppression present in patients with ACLF.

Our study has two important limitations. First, it is an observational investigation looking into associations between KP activity and patients' characteristics or clinical course, and although significant associations may be suggestive, they do not ensure cause-to-effect relationships. Second, some active KP derivatives were not detectable using our untargeted metabolomics workflow (essentially due to sensitivity limitations), and our data are discussed in terms of $\mathrm{KP}$ activity rather than specific Trp-KYN derivatives.

In summary, we show that $\mathrm{KP}$ is activated in patients with $\mathrm{AD}$, especially in those with ACLF, likely as a result of SI and secondary activation of IDO enzymes. We also observed close associations between the KP activity and the presence and/or development of kidney and brain dysfunction/failure, ACLF, impairment in systemic circulatory function, nosocomial bacterial infections, and mortality. TrpKYN derivatives, which are known to have neurotoxic, pro-oxidant, immunosuppressive, endothelial dysfunctional and pro-apoptotic properties, could play an active role in these associations.

Acknowledgment: P.G. is a recipient of an ICREA Academia Award.

\section{REFERENCES}

1) Moreau R, Jalan R, Ginès P, Pavesi M, Angeli P, Cordoba J, et al. Acute-on-chronic liver failure is a distinct syndrome that develops in patients with acute decompensation of cirrhosis. Gastroenterology 2013;144:1426-1437.

2) Bernardi M, Moreau R, Angeli P, Schnabi B, Arroyo V. Mechanisms of decompensation and organ failure in cirrhosis: from peripheral arterial vasodilation to systemic inflammation hypothesis. J Hepatol 2015;63:1272-1284.

3) Trawale JM, Paradis V, Rautou PE, Francoz C, Escolano S, Sallée M, et al. The spectrum of renal lesions in patients with cirrhosis: a clinic-pathological study. Liver Int 2010;30:725-732.

4) Batterworth RF. The liver-brain axis in liver failure: neuroinflammation and encephalopathy. Nat Rev Gastroenterol Hepatol 2013;10:522-528.

5) Guevara M, Baccaro ME, Gómez-Ansón B, Frisoni G, Testa $\mathrm{C}$, Torre $\mathrm{A}$, et al. Cerebral magnetic resonance imaging reveals marked abnormalities of brain tissue density in patients with cirrhosis without overt hepatic encephalopathy. J Hepatol 2011;55:564-573.

6) Arroyo V, Moreau R, Kamath P, Jalan R, Ginés P, Nevens F, et al. Acute-on-chronic liver failure in cirrhosis. Nat Rev Dis Primers 2016;2:16041.

7) Badawy AA. Kynurenine pathway of tryptophan metabolism: regulatory and functional aspects. Int J Tryptophan Res 2017;10:1-20.

8) Cervenka I, Agudelo LZ, Ruas JL. Kynurenines: tryptophan's metabolites in exercise, inflammation, and mental health. Science 2017;357:1-8.

9) Munn DH, Mellor AL. Indoleamine 2,3 dioxygenase and metabolic control of immune responses. Trends Immunol 2013;34:137-143.

10) Wirthgen E, Hoeflich A, Rebl A, Günther J. Kynurenic acid: the Janus-faced role of an immunomodulatory tryptophan metabolite and its link to pathological conditions. Front Immunol 2018;8:1957.

11) Palego L, Betti L, Rossi A, Giannaccini G. Tryptophan biochemistry: structural, nutritional, metabolic and medical aspects in humans. J Amino Acids 2016;2016:8952520.

12) Greco AV, Mingrone G, Favizzi A, Bertuzzi A, Gandolfi A, DeSmet R, et al. Subclinical hepatic encephalopathy: role of tryptophan binding to albumin and the competition with indole-3 acetic acid. J Invest Med 2000;48:274-280.

13) Allison DJ, Ditor DS. The common inflammatory etiology of depression and cognitive impairment: a therapeutic target. J Neuroinflammation 2014;11:151.

14) Gustot T, Fernández J, García E, Morando F, Caraceni P, Alessandria $\mathrm{C}$, et al. Clinical course of acute-on-chronic liver failure syndrome and effects on prognosis. Hepatology 2015;62:243-252.

15) Grønbæk H, Rødgaard-Hansen S, Aagaard NK, Arroyo V, Moestrup SK, Garcia E, et al. Macrophage activation markers predict mortality in patients with liver cirrhosis without or with acute-on-chronic liver failure. J Hepatol 2016;64:813-822.

16) Clària J, Stauber RE, Coenraad MJ, Moreau R, Jalan R, Pavesi $M$, et al. Systemic inflammation in decompensated 
cirrhosis: characterization and role in acute-on-chronic liver failure. Hepatology 2016;64:1249-1264.

17) Fernández J, Acevedo J, Weist R, Gustot T, Amoros A, Deulofeu $\mathrm{C}$, et al. Bacterial and fungal infections in acute-on-chronic liver failure: prevalence, characteristics and impact on prognosis. Gut 2018;67:1870-1880.

18) Boudah S, Olivier MF, Aros-Calt S, Oliveira L, Fenaille F, Tabet JC, et al. Annotation of the human serum metabolome by coupling three liquid chromatography methods to high-resolution mass spectrometry. J Chromatogr B Analyt Technol Biomed Life Sci 2014;966:34-47.

19) Dunn WB, Broadhurst D, Begley P, Zelena E, Francis-McIntyre $\mathrm{S}$, Anderson N, et al. Procedures for large-scale metabolic profiling of serum and plasma using gas chromatography and liquid chromatography coupled to mass spectrometry. Nat Protoc 2011;6:1060-1083.

20) Gostner JM, Becker K, Kofler H, Strasser B, Fuchs D. Tryptophan metabolism in allergic disorders. Int Arch Allergy Immunol 2016;169:203-215.

21) González A, Varo N, Alegre E, Díaz A, Melero I. Immunosuppression routed via the kynurenine pathway: a biochemical and pathophysiologic approach. Adv Clin Chem 2008;45:155-197.

22) Asghar K, Brain J, Palmer JM, Douglass S, Naemi FMA, O'Boyle G, et al. Potential role of indoleamine 2,3-dioxygenase in primary biliary cirrhosis. Oncol Lett 2017;14:5497-5504.

23) Yang $R$, Gao N, Chang $Q$, Meng $X$, Wang W. The role of IDO, IL-10 and TGF- $\beta$ in the HCV-associated chronic hepatitis, liver cirrhosis, and hepatocellular carcinoma. J Med Virol 2019;91:265-271.

24) Batshaw W, Robinson MB, Hyland K, Djali S, Heyes MP. Quinolinic acid in children with congenital hyperammonemia. Ann Neurol 1993;34:676-681.

25) Basile AS, Saito K, Al-Mardini H, Record CO, Hughes RD, Harrison P, et al. The relationship between plasma and brain quinolinic acid levels and the severity of hepatic encephalopathy. Gastroenterology 1995;108:818-823.

26) Mohib K, Wang S, Guan Q Mellor AL, Sun H, Du C, et al. Indoleamine 2,3-dioxygenase expression promotes renal ischemia-reperfusion injury. Am J Physiol Renal Physiol 2008;295:F226-F234.

27) Vecsei L, Szalady L, Fülop F, Toldi J. Kynurenines in the CNS: recent advances and new questions. Nat Rev Drug Discov 2013;12:64-82.

28) Aldridge DR, Tranah EJ, Shawcross DL. Pathogenesis of hepatic encephalopathy: role of ammonia and systemic inflammation. J Clin Exp Hepatol 2015;5:S7-S20.
29) Wang Y, Liu H, McKenzie G, Witting PK, Stasch JP, Hahan M, et al. Kynurenine is a novel endothelium-derived relaxing factor produced during inflammation. Nat Med 2010;16:279-285.

30) Jung ID, Lee MG, Chang JH, Lee JS, Jeong YI, Lee CM, et al. Blockade of indoleamine 2,3-dioxygenase protects mice against lypopolysaccharide-induced endotoxic shock. J Immunol 2009;182:3146-3154.

31) Wilson JRA, Morandi A, Girard TD, Thompson JL, Boomershine CS, Shintani AK, et al. The association of kynurenine pathway of tryptophan metabolism with acute brain dysfunction during critical illness. Crit Care Med 2012;40:835-841.

32) Pellegrin K, Neurauter G, Wirleitner B, Fleming AW, Peterson VM, Fuchs D. Enhanced enzymatic degradation of tryptophan by indoleamine 2-3-dioxygenase contributes to the tryptophandeficient state seen after major trauma. Shock 2005;23:209-215.

33) Skouras C, Zeng X, Binnie M, Homer NZ, Murray TB, Robertson D, et al. Increased levels of 3-hydroxykinurenine parallel disease severity in human acute pancreatitis. Sci Rep 2016;6:33951.

34) Huttunen R, Syrjänen J, Aittonirmi J, Oja SS, Raitala A, Laine $\mathrm{J}$, et al. High activity of indoleamine 2,3 dioxygenase enzyme predicts disease severity and case fatality in bacteremic patients. Shock 2010;33:149-154.

35) Hoshi M, Osawa Y, Ito H, Ohtaki H, Ando T, Takamatsu M, et al. Blockade of indoleamine 2,3-dioxygenase reduces mortality from peritonitis and sepsis in mice by regulating functions of CD11b+ peritoneal cells. Infect Immun 2014;82:4487-4495.

36) Mole DJ, Webster SP, Uins I, Zheng X, Binnie M, Wilson K, et al. Kynurenin-3-monooxigenase inhibition prevents multiple organ failure in rodent models of acute pancreatitis. Nat Med 2016;22:202-209.

37) Mandy Y, Vecsey L. The kynurenin system and immuneregulation. J Neural Transm 2011;119:197-209.

38) Wirthgen E, Hoeflich A. Endotoxin induced tryptophan degradation along the kynurenin pathway: the role of Indolamine 2,3-dioxygenase and aryl hydrocarbon receptor-mediated immunosuppressive effects in endotoxin tolerance and cancer and its implications for immune paralysis. J Amino Acids 2015;2015: 973548.

Author names in bold designate shared co-first authorship.

\section{Supporting Information}

Additional Supporting Information may be found at onlinelibrary.wiley.com/doi/10.1002/hep.30363/suppinfo. 